\title{
The Effective Application of Fragmented Learning Guided by Constructivism
}

\author{
Ying Jiang \\ English Department of Humanities and Social Sciences, Heilongjiang Bayi Agricultural University, Daqing, China \\ Email address: \\ happyying101@163.com \\ To cite this article: \\ Ying Jiang. The Effective Application of Fragmented Learning Guided by Constructivism. International Journal of Education, Culture and \\ Society. Vol. 3, No. 1, 2018, pp. 10-13. doi: 10.11648/j.ijecs.20180301.13
}

Received: March 5, 2018; Accepted: March 23, 2018; Published: April 13, 2018

\begin{abstract}
The rapid development of science and technology and the growing information dissemination have enabled people to broaden the channels for acquiring knowledge. With the continuous advancement of Internet technology, a more intimate and simpler learning method has emerged quietly, which has also triggered profound changes in traditional learning methods. This paper aims to combine relatively independent fragmented learning with traditional learning methods by fully analyzing the fragmented learning. Guided by the theory of constructivism, the paper analyzes the shortcomings of fragmented learning and discuses how to make use of its advantages in the era of information technology. It is concluded that combined the characteristics of fragmented learning, it will supplement the traditional learning methods through learners' conscious integration of knowledge structure.
\end{abstract}

Keywords: Constructivism, Fragmented Learning, Application

\section{Introduction}

On August 4, 2017, the 40th Statistical Report on the Internet Development in China released by China Internet Network Information Center (CNNIC) showed that as of June 2017, the number of netizens in China reached 751 million, an increase of $2.7 \%$ half a year. Internet penetration rate was $54.3 \%$. Among them, the number of online education users reached 144 million. With the popularization of mobile intelligent terminals and the improvement of network infrastructure, profound changes have taken place in the content and methods of learning. Fragmented learning has emerged. However, this novel and flexible learning method also has some drawbacks. How to give full play to its advantages in the information age and how to effectively optimize the learning experience are the issues to be explored in this article.

Fragmented learning has strong technical support. Cloud computing technology can aggregate massive resources into huge storage and has tremendous computing power. It can aggregate and co-operate large amounts of information and processor resources stored on personal computers, mobile phones, and other mobile devices. The $4 \mathrm{G}$ network quickly links fragmented learning resources stored in the cloud to various mobile devices and enables synchronous interaction. This allows a variety of fragmented learning applications to help learners more autonomous and more convenient to conduct fragmented learning.

Looking at the history of the development of educational technology, every step of its development includes the integration and application of the latest technological achievements. In the Internet era, the speed of information dissemination and form of its presentation have all changed radically. How to locate the required knowledge from massive information is an essential quality for modern learners. The main goal of this study is to take advantage of the fragmented learning in the era of information technology, to effectively optimize the learning experience, and to propose practical advice in combination with traditional learning methods. It first elaborates the content of constructivism learning theory, and analyzes the connotation, advantages and disadvantages of fragmented learning, and finally proposes effective utilization of fragmented learning.

In recent years, the rise of fragmented learning has attracted the attention of many researchers. The Learning Lab developed by Stanford University in the United States developed a mobile learning model for foreign language learning to train learners' language skills. The study showed 
that the learners' attention in fragmented learning is fragmented. Learning brings a variety of new contextual relationships. China's research on fragmented learning started late, but it has developed rapidly and has achieved certain results. Prof. Zhu Zhiting [1] pointed out that fragmented learning is a new development in China's education informatization. There are also theoretical discussions on fragmented learning, including the discussion of the advantages and disadvantages of fragmented learning, such as Wang Chengbo [2] and Huang Jianfeng [3]. In practice, it tends to study how information resources are fragmented. For example, Wang Mi [4] analyzes content decomposition design strategies for micro-video courses, and constructs a "heartbeat" design model of teaching micro-video based on the SMCR propagation model and ARCS motivation model, providing better help for designers and educators of microvideo course content. At present, the research focus of fragmented learning is the use of platform or media integration to deliver fragmented information. Yu Haiyan [5] believes that the MOODLE platform based on social constructivism design should combine system design, functional blocks and independent small blocks, media attributes and content. Four aspects of convergence, microlearning and other aspects are optimized to improve the effectiveness of the MOODLE curriculum platform.

\section{Constructivism Learning Theory}

Constructivism is an important branch of cognitive psychology. In the 1960s, J. Piaget, a famous psychologist in Switzerland, studied the characteristics of children's cognitive development through the interaction between internal and external factors. In the process of environmental interaction, the understanding of the outside world is gradually built so that individual's cognitive structure can be developed. Later, many psychologists and educators, such as Vygot-sky, Ausubel, Bruner, reviewed the nature and development conditions of cognitive structure, the influence of human social environment on psychological development, and the important role of individual initiative in the process of constructing cognitive structure, enriching and developing the constructivism theory. With the advent of the information technology revolution and the extensive application of the Internet, constructivist learning theory plays an increasingly important role.

Constructivism comes from the cognitive development of children. Because individual's cognitive development is closely related to the learning process, the use of constructivism can explain the cognitive law of human learning process well. This paper mainly introduces the contents of constructivist learning theory from the meaning of learning and the learning methods.

First of all, learning is not a simple one-way input of information from the outside to the inside, but a two-way interaction between the new information and the learner's original knowledge experience, that is, the process of interaction between the learner and the learning environment.
There are three points in this process that need to be noticed. First, learning is not equivalent to the direct transmission of information. Instead, it constructs its own cognitive system by understanding the representation of things and integrates with learners' original knowledge and experience to form meaning of new information, and transform or reorganize the original experience. Second, meaning construction is the process of learner's interaction with the environment and the process of meaning construction through the interpersonal collaboration activities. Therefore, constructivist learning theory holds that "situation", "collaboration", "conversation" and "meaning construction" are four elements of a learning environment. Third, the exploration of meaning by learners, that is, the exploration of the law of things is a process of active exploration and cannot be replaced by others. From the above, it is believed that how much knowledge can be absorbed is not determined by external factors, but by the learners themselves who have completed how much meaning construction.

Second, constructivism advocates a learner-centered approach to learning under the guidance of teachers. Students should be the active constructors of meaning, complete the receiving and processing, reforming and reorganizing of information. Therefore, in the process of learning, students should explore the meaning of knowledge and complete the construction. In the course of constructing meaning, learners should take the initiative to collect and analyze relevant information and materials, make various assumptions about the learning problems and try hard to verify it. Try to connect the things reflected in the current study with the things you already know and think carefully about the connection. To achieve efficient meaning construction, on the one hand, students should learn to relate and think about the relationship between new meaning and existing knowledge. They should also deepen their understanding in the collaborative learning of exchange and discussion and realize the meaning construction of high-quality.

\section{Fragmented Learning}

\subsection{The Connotation of Fragmented Learning}

Today, the rapid development of Internet information technology, and update of mobile intelligent terminals provide good technical conditions for the popularity of fragmented learning. Although there has been no universally accepted concept of fragmented learning in recent years, its rich connotations can be summarized as follows:

Fragmented learning content. Different from the whole knowledge system, fragmented learning decomposes the content into fragmented knowledge fragments, forming small knowledge points with fragments as a unit. There is no definite logical structure between knowledge points and the way of presentation is more diversified.

Fragmentation learning time. Fast-paced modern life forms a fragmented, patchwork form of time. Compared with the chunk of learning time, short, free, fragmented time is more 
flexible, from a few minutes to no more than 15 minutes, Learning often can be seen while waiting for the car, taking a bus, lining up, or intervals.

Fragmented learning environment. Formal learning environments are generally quiet, but fragmented learning takes place in relatively noisy or casual environments, leaving learners relaxed.

Fragmented thinking. There are two kinds of understanding. In terms of learners, Prof. Popkewitz thinks that "fragmentation" in fragmented thinking embodies a process of "de-naturalization", that is, in the "Internet +" era, learners need to break conventional thinking paradigm, to take a new way of thinking suited to their needs. It emphasizes the creativity of learners. In terms of information providers, according to learners' needs, they divide the whole information into information fragments and even guide the needs of learners.

From the above characteristics of fragmented learning, it is concluded that fragmented learning is a process of learning fragmented knowledge according to learners' needs, interests, and ways of thinking without limitation of time and space, and may form a creative update to the existing knowledge structure.

\subsection{Drawbacks of Fragmented Learning}

Long-term fragmented learning will make learners less discriminating. A notable feature of the Internet age is the era of big data flows in the hyper-mobile era where a large amount of fake, incomplete and inaccurate fragmented information in poor quality generates information pollution. Without discriminating, the valuable information and knowledge is overwhelmed, learners' insight will also degenerate and shrink.

Fragmentation of learning lacks of deep thinking easily. In order to maintain the freshness and popularity of information, fragmented information has the characteristics of "short", "flat" and "fast". Although this rapid replacement of information ensures timeliness, and meets the needs of learners, they cannot concentrate on in-depth analysis of thinking, cannot understand the principles behind and the interrelationships between things, cannot learning intensively around a single subject of knowledge, because the complex information is posted simplified, and knowledge is frequently switched.

Fragmented learning interferes with systematic knowledge collation. A notable feature of fragmented learning is cutting the knowledge structure into fragmented knowledge points or information, making it easy for learners to learn anytime, anywhere. This way of learning makes people feel that they receive information every day, but the old information is constantly covered by new information, and the knowledge without internalization of analysis is easily forgotten. In addition, the scattered knowledge makes learners thinking one-sided and isolated. Thinking without logical reasoning and deduction will lead to the lack of logical thinking over time, which is not conducive to the development of the overall logical thinking of learners.

\subsection{The Effective Use of Fragmented Learning}

Compared with traditional learning, fragmented learning is a learning revolution with the characteristics of the times. It undoubtedly changes people's learning habits in a subtle way, and also plays a role in lifelong learning with the times. How to avoid weaknesses, enable learners to effectively use fragmented learning, combined with constructivism learning theory, some useful ideas are presented.

i. Clear learning purpose and improve the ability of information identification. Constructivism believes that learning is not a mechanical passive acceptance of knowledge, but to take the initiative to analyze, to identify, and then integrated into their existing knowledge system. Therefore, learners should improve the processing capabilities of fragmented information. If they do not want to be overwhelmed by vast amounts of information, learners should combine their own interests and purposes, to improve rational thinking and judgment. They need clear purpose, take the initiative to filter unrelated information, and improve the efficiency of access to knowledge.

ii. Develop advanced thinking ability to solve problems. Although in the short run the tendency of fragmented learning content not only accords with the operation characteristics of information media, but also caters for learners' habits. Short-term browsing or memory of knowledge cannot realize the construction and effective use of meaning. The construction of knowledge is accomplished through the interaction of new and old knowledge experiences. Learning on the one hand is to acquire knowledge; the other important role is to solve the problem. It requires a long-term training of advanced thinking or logical thinking in learning. Combined with problems and purposes, learners need to obtain relevant information and extract the original knowledge to explain the phenomenon, build assumptions and test through a certain way. This connection and reflection is not only the key to constructivism learning, but also the ideal way for learners to construct knowledge and cultivate their ability to solve problems.

iii. Integrate the knowledge structure according to learning needs. Because of the fast update of fragmented learning knowledge, it has the advantage of cutting the knowledge frontier for learners. If used well, it will be a useful complement to individual's knowledge structure. Therefore, learners should design a structured knowledge system according to their own learning needs, which helps clarify the fragmented information. First of all, clear the theme of knowledge, under which how many branches can support, and what knowledge points can be contained in each branch. What knowledge points are relatively complete, and what knowledge needs to be further updated or supplemented? Therefore, the top-down fragmentation of knowledge through effective segmentation and organization can also help learners to understand the linkages of fragmented information from a simple perspective. By reorganizing, this method reproduces the overall structure of knowledge, easy 
to understand and remember.

\section{Conclusion}

In short, fragmented learning is an inevitable product of people' entry into the era of information technology, and an unavoidable new way of learning. Any knowledge has always been a complete whole, but because of the changes of the times and ways of learning, the presentation of knowledge adopts a new way, the whole into a part, but after all, to return to the whole. This requires that learners organize and classify the knowledge gained through fragmented learning to find out the relevance and then internalize it into a holistic internal knowledge. Only in this way can the fragmented learning become an effective complement to the traditional learning methods in order to truly bring into play the maximum effectiveness of fragmented learning.

\section{Acknowledgements}

This work is supported by The Key Research Programs for The Economic and Social Development in Heilongjiang Province in 2017 (Foreign Language Discipline) (No. WY2017041-B).

\section{References}

[1] Zhu Zhiting. The Latest Developments of Education Informatization: International Observations and Domestic Trends. Modern Distance Education Research, 2012, (3): 313 .

[2] Wang Chengbo, Li Xiaoping, Zhao Fengnian, Zhang Lin. Research on Fragmented Learning in the Era of Big Data. Electronic Education Research, 2015 (10): 26-30.

[3] Huang Jianfeng. Fragmented Learning: A New Style of Learning Based on "Internet+". Education Exploration, 2016 (12), 115-119.
[4] Wang Mi. Content Design for Micro-video Courses in the Fragmented Learning Era. East China Normal University, 2013.

[5] Yu Haiyan. Fragmented Learning: A New Style of Learning Based on "Internet+". Theory and Practice of Education, 2016 (6), 43-44.

[6] T. M. Duffy, and D. H. Jonassen. Constructivism: New Implications for Instructional Technology. Educational Technology Publications, 1992, 31 (5): 7-12.

[7] Klahr D, Nigam M. The equivalence of learning paths in early science instruction: Effects of direct instruction and discovery learning. Psychological Science, 2004 (15): 661-667.

[8] Henk G. Schmidt, Sofie M. M. Loyens, Tamara van Gog, and Fred Paas. Problem-Based Learning is Compatible with Human Cognitive Architecture: Commentary on Kirschner, Sweller, and Clark. Educational Psychologist, 2007 (42), 9197.

[9] Sigmund Tobias, and T. M. Duffy. Constructivist Instruction: Success or Failure. New York: Routledge, 2009, 47-188.

[10] Huang Jianfeng. Research on Fragmentation Learning Strategy Based on "Internet Plus":The Evolution from "Fragment" to "Integrality". Electrical Education Research, 2017 (8), 78-82.

[11] Wang Chengbo, Li Xiaoping, Zhao Fengnian, etc. Research on Fragmented Learning in Big Data Era. Electrical Education Research, 2015 (10), 26-30.

[12] Wang Zhuli. New Constructivism: Learning Theory in the Internet Age. Journal of Distance Education, 2011 (2), 11-18.

[13] Wang Zhuli. How to Make Use of Fragmented Learning. China Information Technology Education, 2016 (12), 4-10.

[14] Zhao Jing. "Fragmentation" of Thinking and Education --- An Interview with Professor Thomas Pockowitz. Global Education Outlook, 2012 (10), 3-7.

[15] Zhang Keyong, Li Yujia, and Yang Xue. The Research of Cognitive Disorder in Network Fragmented Learning. Modern Educational Technology, 2015 (2), pp88-94. 